Ensayo

\title{
Breve reseña de los aspectos taxonómicos y nomenclaturales actuales del género Cinchona (Rubiaceae-Cinchoneae)
}

\section{A brief outline on current taxonomical and nomenclatural aspects of the genus Cinchona (Rubiaceae-Cinchoneae)}

\author{
Gerardo A. Aymard C. ${ }^{1,2}$ \\ ${ }^{1}$ Compensation International Progress S. A. - Ciprogress Greenlife, Bogotá, D. C. Colombia \\ ${ }^{2}$ UNELLEZ-Guanare, Programa de Ciencias del Agro y el Mar, Herbario Universitario (PORT), Mesa de Cavacas, estado Portuguesa, Venezuela
}

\begin{abstract}
Y de allí surgió la fiebre, como fiebre del oro; la quina anaranjada, la quina roja, giraban en la mente de los cazadores de fortunas. Se inició la odisea, la anuencia, los bosques empezaron a ser violentados por los machetes sedientos de oro... La fiebre corría y la quina paradójicamente la encendía.
\end{abstract}

Las cortezas y raíces de las diferentes especies de los árboles del género Cinchona L. (entre ellas, C. calisaya Wedd., C. officinalis L. y C. pubescens Vahl), han sido ampliamente utilizadas por su gran virtud febrífuga, igualmente para las arritmias cardiacas, los calambres musculares, resfriados, indigestión, fibrilación auricular, tónico eupéptico, catarros, acelerante del parto y como tónico capilar (Pérez Arbelaez, 1947; García-Barriga, 1975; Loayza-O, et al., 2010). La eficacia curativa de la corteza de los árboles de Cinchona, conocidos como "Quina-quina" (amarilla, anaranjada, roja), "Cascarilla", "Yara chucchu", "Cara chucchu" en Ecuador y Perú (La Condamine, 1738, 1745), precede a los tiempos colombinos. En Colombia se conoce como Cascarilla", "Funa", "Azuceno colorado", "Cascarillo gallinazo" "Cascarilla amarga", "Quina amarilla", "Quina terciopelo", "Quina calisaya" "Caquetá", "Quina tunita", "Quina anaranjada" y "Tunita" (Mendoza, et al., 2004). Los pueblos originarios de la cordillera de los Andes también la utilizaban para el tratamiento de las que serían llamadas fiebres terciarias y cuaternarias (Jaramillo, 1951). Se tiene conocimiento de que en la región de "Los Andaquies" (actual departamento del Caquetá), los “Andaki" eran reconocidos por sus sabidurías acerca de la "Quina" y de numerosas plantas medicinales (Albis, 1855). Al presente, el lenguaje "Andaki" se considera extinto (Coronas-Urzua, 1995); sin embargo, y afortunadamente, José Celestino Mutis elaboró notas bibliográficas del vocabulario "Andaki" en 1787 (Freide, 1946a, b). Esta información fue mejorada por Rivet (1924) y Coronas-Urzua (1995), respectivamente.
Posteriormente, con el arribo de los españoles, la "Quina" fue "descubierta" por estos como el más efectivo remedio para los terribles estados febriles que los aquejaban (en muchos de los casos mortales). Como consecuencia, su uso se difundió por todas sus colonias del "nuevo mundo". De los primeros testimonios acerca de la muerte de numerosos soldados debilitados por las calenturas intermitentes, y otras calamidades tropicales, se encuentran bien documentadas en las crónicas escritas por fray G. de Carvajal, F. Vázquez y P. de Almesto (Carvajal, 1848; Simón, 1861). Los afamados cronistas de los primeros dos viajes desde los Andes hasta la desembocadura del río Amazonas, capitaneados por Francisco de Orellana entre 1541 y 1542, y Pedro de Ursúa y L. de Aguirre entre 1560 y 1561 respetivamente.

El principio del siglo XVI, marcó el inicio de la interesante y fascinante historia de la corteza de la "quina", la cual continúa hasta nuestros días a través de numerosos acontecimientos notables (Soares-Ferreira-Jr, et al., 2012). Destacándose, el nombre de "corteza de los Jesuitas" (Prendergast, 2001) y el monopolio de este producto por parte de la corona española en sus virreinatos a través de la ruta andina de las quinas. La cual partía desde la porción central de Bolivia hasta los límites de Colombia con Venezuela en el departamento del Norte del Santander (Crawford,

Correspondencia:

Gerardo A. Aymard C., gaymard@ciprogress.com

Recibido: 30 de octubre de 2019

Aceptado: 31 de octubre de 2019

Editor: Elizabeth Castañeda 
2016; ver figura1). Las cifras más conservadoras indican que durante los siglos XVII y XVIII se exportaban a Europa aproximadamente medio millón de kilos de cortezas por año (Roersch van der Hoogte \& Pieters, 2015). La presente reseña no pretende ser un ensayo del tema, sin embargo, para información en detalle de la historia de la "quina", se recomienda la serie "El Arcano de la Quina", publicada por José Celestino Mutis en el Papel Periódico de Santafé de Bogotá, entre el 10 de Mayo de 1793 al 7 de Febrero de 1794 (Kirkbride Jr., 1982). Igualmente, las ocho publicaciones contenidas en este suplemento, y adicionalmente las extraordinarias contribuciones de Sandoval-B. y Echendia-C. (1987), Rodríguez-Nozal (1993), Díaz-Piedrahita (2010) y Crawford (2016).

El género Cinchona y su especie tipo (C. officinalis L.) fue descrito por C. Linnaeus $(1753 ; 172)$, basándose en la descripciones e ilustraciones elaboradas por el militar, matemático y geógrafo francés Charles M. de La Condamine (un miembro de la expedición francesa, para medir la longitud de un grado de meridiano terrestre a las proximidades del Ecuador en 1735), de árboles observados en 1737 en la montaña de Caxanuma, ubicada ca. $15 \mathrm{~km}$ al sur de la villa de Malacatos, Loja, Ecuador (La Condamine, 1738; Crawford, 2016). La publicación de La Condamine, está acompañada de varias hojas, las cuales fueron de gran ayuda para estudios posteriores, y al presente estas se conservan en buen estado (Andersson, 1994). Paradójicamente, C. officinalis L., es el taxón con las menores cantidades de quinina (Nair, 2010), para esta época, la especie más utilizada para tratar la malaria fue $C$. pubescens Vahl de la región de Loja, Ecuador. La cual fue descrita en 1790 por el botánico danés Martin Vahl, a través del material obtenido por Antoine Laurent de Jussieu (el botánico de la expedición meridiano), aparentemente en el mismo lugar (Nudo de Caxanuma, Ecuador) que las muestras de La Condamine (Andersson, 1998). Posteriormente, durante los siglos XVIII y XIX, nuevas especies fueron descritas por J. C. Mutis (1793), H. Ruíz-López y J. A. Pavón y Jiménez $(1799,1802)$ y A. von Humboldt y A. Bonpland $(1805,1808)$, respectivamente. Entre 1804-1805 sucedió un hecho inusual de los muchos en la historia de la "quina"; el botánico e ilustrador navarro J. J. Tafalla y Nabasques herborizó intensamente al sur del Ecuador y norte del Perú. Los excicattae le fueron cedidos al botánico extremeño José Antonio Pavón y Jiménez, quien describió nuevas especies de Cinchona, las cuales nunca publicó: al contrario, vendió el manuscrito y los excicattae. Posteriormente, A. B. Lambert en 1821, A. P. de Candolle en 1829 y J. Lindley en 1938 publicaron varias de estas especies. Sin embargo, J. E. Howard adquirió el manuscrito de Pavón y le añadió ilustraciones, y entre 1859 y 1862 culminó este trabajo (Andersson, 1998). Aparte de las contribuciones de Lambert, De Candolle y Lindley, el género fue estudiado por H. A. Weddell en 1849, H. Karsten en 1859 y O. Kuntze en 1878 , respectivamente. Contribuciones que, de acuerdo con

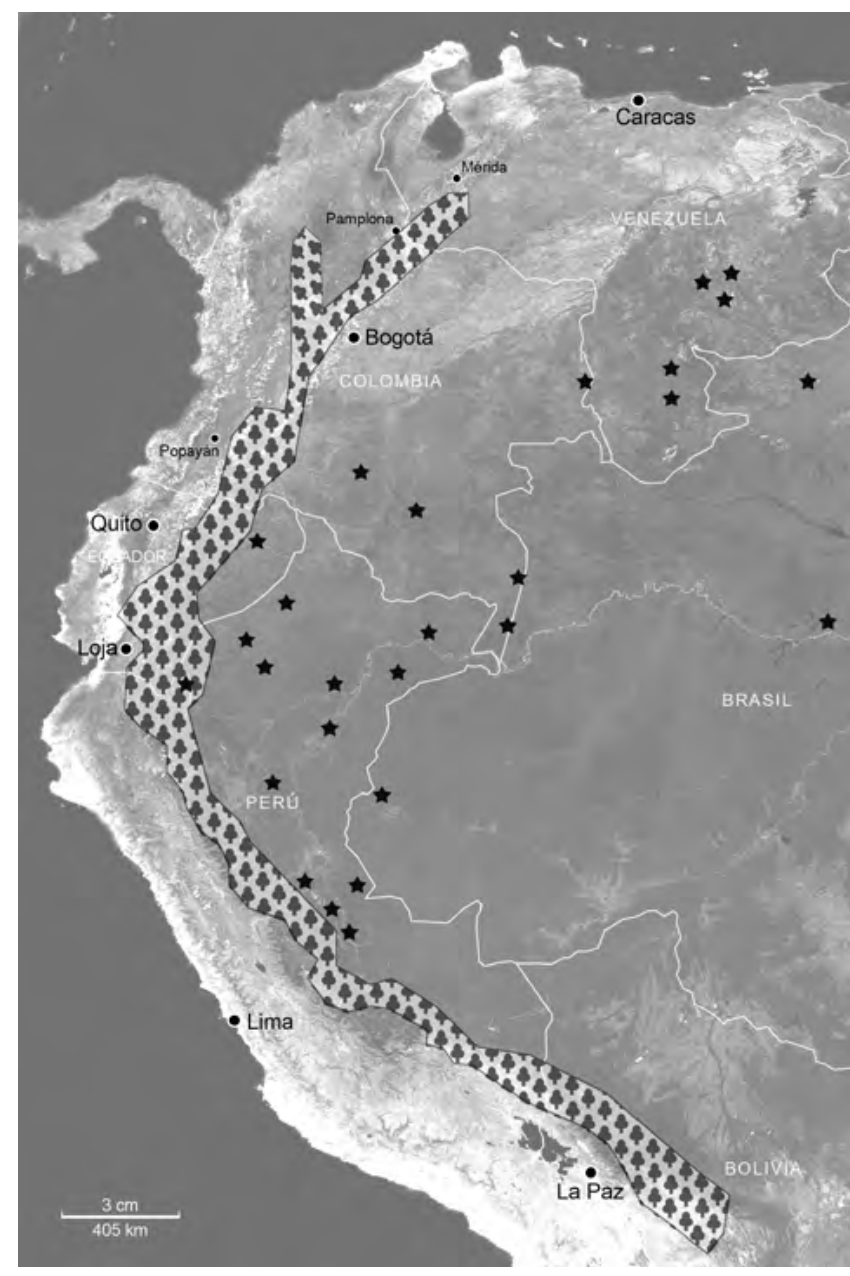

Figura 1. La ruta de la quina durante los siglos XVII y XVIII, y la distribución geográfica de Cinchonopsis amazonica (estrella negra), elaborado por Carlos Méndez. Noviembre, 2019.

Andersson (1998), no mostraron progresos significativos con respecto a una monografía formal del mismo. El aporte de G. K. W. H. Karsten al conocimiento del género se centra en sus exploraciones botánicas durante 12 años en los territorios de las noveles repúblicas de Venezuela (1844--1847 y 1848--1852), Colombia y Ecuador entre 1852--1856 (Röhl, 1944). Los resultados de sus trabajos de campo los publicó en dos volúmenes de su magnífica obra titulada: Florae Columbiae: Tomus primus (1959) y Tomus secundus (1862). En el Tomus primus, se hace referencia al género Cinchona a través de sus extraordinarias reseñas e iconografías (Figura 2), destaca la descripción de la nueva especie Cinchona barbacoensis H. Karst. Desafortunadamente, esta información es poco a nada citada por los quinólogos contemporáneos. En la Tabla 1 se sintetiza su trabajo acerca de las quinas del noreste de Sudamérica.

Pasaron más de 100 años para que Cinchona y sus géneros afines fuesen debidamente organizados dentro de la tribu Cinchoneae (Andersson \& Persson, 1991; Andersson, 1995, 1997). En 1998, se elaboró la monografía 


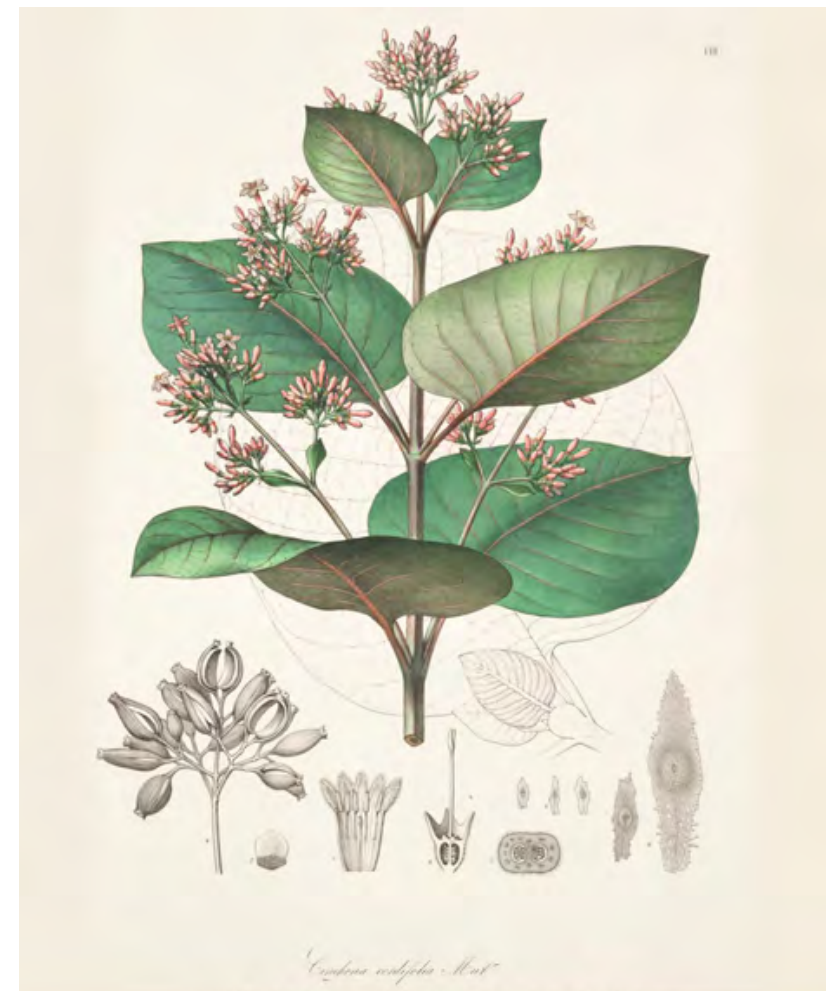

CINCHONA CORDIFOLIA MUT. HUMB.

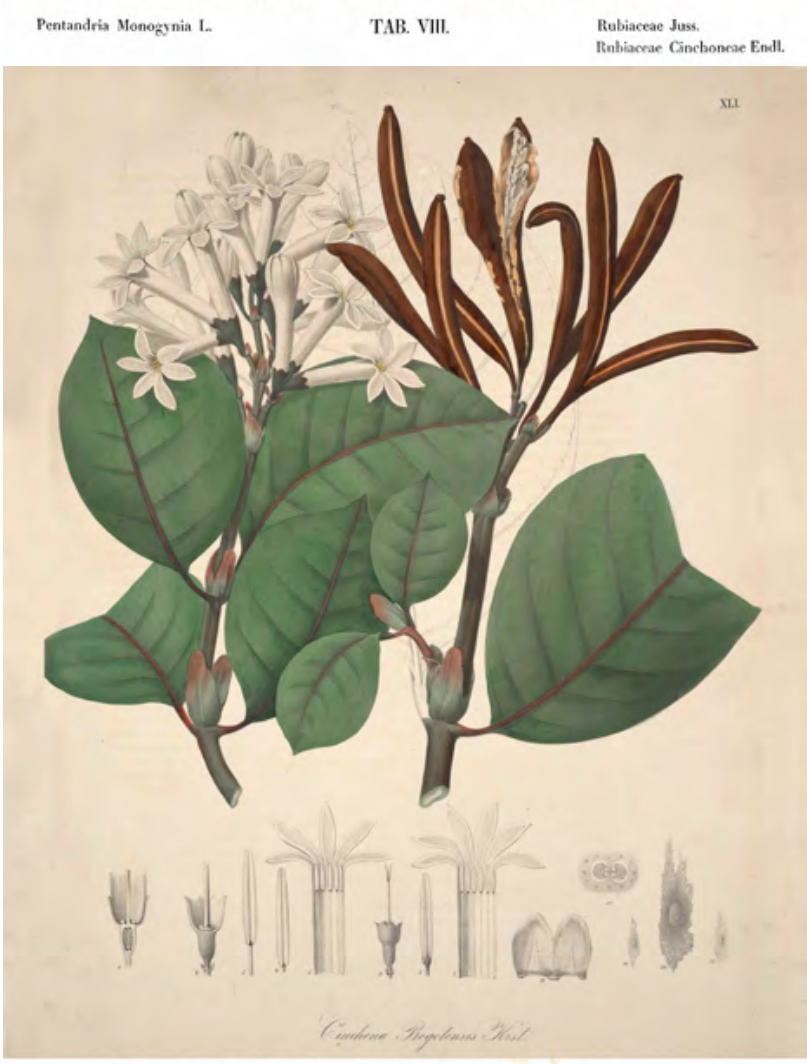

CINCHONA BOGOTENSIS KRST.

Pentandria Monogynia L. TAB. XLI. Rubiaceac Juss.

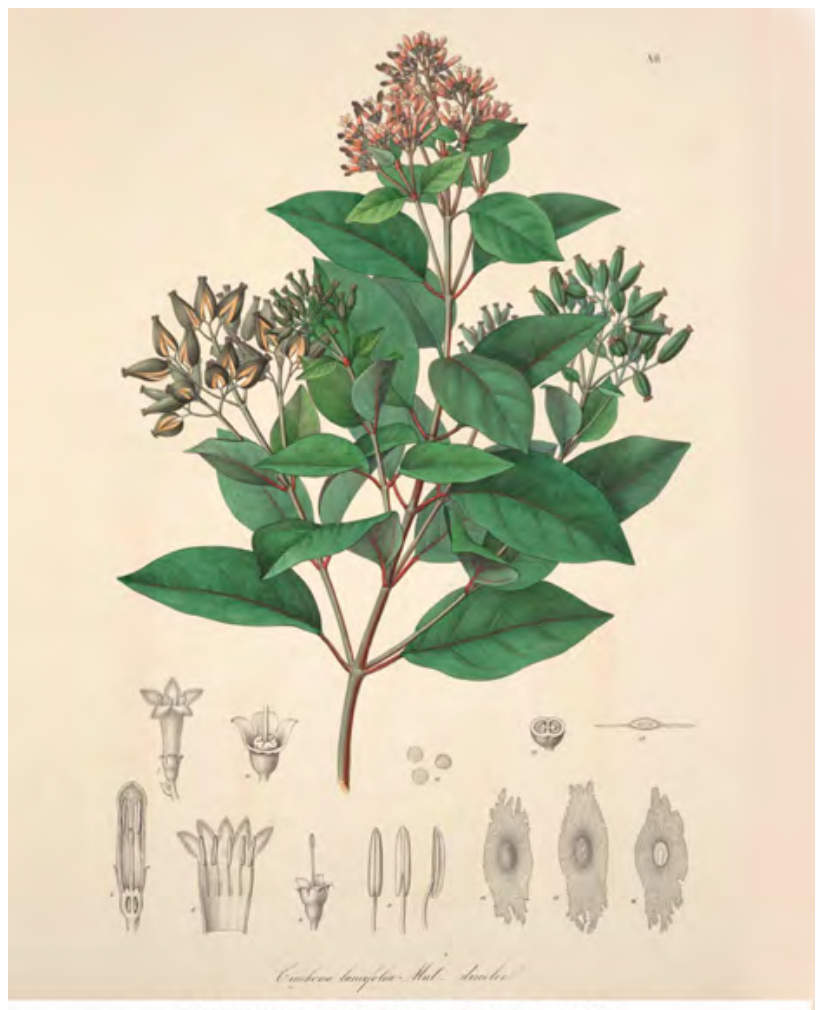

CINCHONA LANCIFOLIA MUT. HUMB.

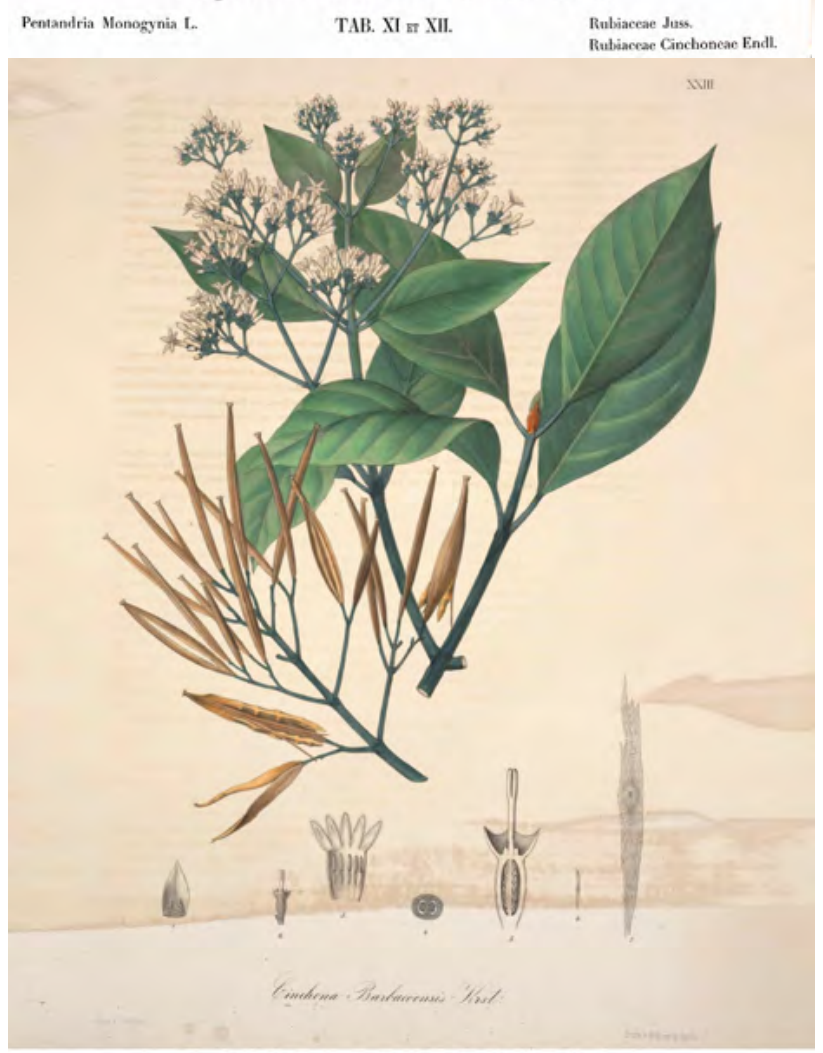

CINCHONA BARBACOENSIS KRST.

Peentandria Monogynis Limo. TAB. XXIII. Rubiaceac Jase

Figura 2. Cuatro láminas de la muestra iconográfica del género Cinchona adjunta en la obra: Florae Columbiae, Tomus primus (Karsten, 1959). 
Tabla 1. Especies del género Cinchona enumeradas por Hermann Karsten en 1859 (columna izquierda), nombres actuales (columna derecha).

\begin{tabular}{|c|c|}
\hline Cinchona barbacoensis H. Karst. & = especie válida \\
\hline C. bogotensis H. Karst. & $=$ sin. de Ladenbergia heterophylla (Wedd.) Standl. \\
\hline C. cordifolia Mutis & $=$ sin. de $C$. pubescens Vahl \\
\hline C. cordifolia var. peruviana Howard & $=$ nombre inválido \\
\hline C. corymbosa H. Karst. & $=$ sin. de $C$. pitayensis (Wedd.) Wedd. \\
\hline C. heterocarpa H. Karst. & $=$ sin. de Ladenbergia oblongifolia (Humb. ex Mutis) L. Andersson \\
\hline C. heterocarpa var. bogotensis & $=$ no publicada \\
\hline C. henleana H. Karst. & $=$ sin de Ladenbergia muzonensis (Goudot) Standl. \\
\hline C. lancifolia Mutis & $=$ especie valida \\
\hline C. lancifolia var. discolor H. Karst. & $=$ sin. de $C$. lancifolia Mutis \\
\hline C. undata (Klotzsch) H. Karst. & $=$ sin. de Ladenbergia undata Klotzsch \\
\hline C. macrocarpa Vahl & $=$ sin. de Landenbergia macrocarpa (Vahl) Klotzsch \\
\hline C. moritziana (Klotzsch) H. Karst. & $=$ sin. de Ladenbergia moritziana Klotzsch \\
\hline C. macrophylla H. Karst. & $=$ sin. de Remijia macrophylla (H. Karst.) Benth. \& Hook. f. ex Flueck. \\
\hline C. pedunculata H. Karst. & $=$ sin. de Ciliosemina pedunculata (H. Karst.) Antonelli \\
\hline C. prismatostylis H. Karst. & $=$ sin. de Ladenbergia macrocarpa (Vahl) Klotzsch \\
\hline C. trianae H. Karst. & $=$ sin de C. pitayensis (Wedd.) Wedd. \\
\hline C. tucujensis H. Karst & $=$ sin. de C. pubescens Vahl \\
\hline
\end{tabular}

del género, para esta fecha, 330 nombres fueron considerados en la revisión del mismo (Andersson, 1998). En esta contribución, fue de gran ayuda los numerosos exsiccatae colectados durante los trabajos de la misión norteamericana de Cinchona en América entre 1940 y 1945 (Steere, 1945). Una actividad originada a partir de la invasión de los japoneses a los países del Sudeste Asiático, durante la segunda guerra mundial (Hodge, 1948). Estas exploraciones permitieron el arribo de distinguidos botánicos al continente americano (i.g. R. Fosberg y J. A. Steyermark). Es de destacar, que la misión norteamericana ha sido considerada como una expresión más del colonialismo científico de las grandes potencias (Cuvi, 2011).

Actualmente, Cinchona es un género reducido a 24 especies distribuidas en Costa Rica, Panamá, la Cordillera de la Costa en Venezuela, y en los Andes, desde Venezuela hasta el centro de Bolivia, ausente en Brasil, las Guayanas, México y el Cono Sur (Ulloa-Ulloa, et al., 2017, 2018 onwards; ver tabla 2). La última especie descrita a la fecha es $C$. anderssonii Maldonado, un arbusto a pequeño árbol, al presente solamente conocido en bosques montanos (2200$2600 \mathrm{~m}$ ) de la región de las Yungas, Bolivia (Maldonado, et al., 2017a). Las regiones montañosas de los sectores central y noreste de cordillera andina (hasta $3.300 \mathrm{~m}$ ) representan los hábitats dominantes del género (Antonelli, et al., 2009), Ecuador y Perú poseen la mayor cantidad de especies, con 14 y 18, respectivamente. Varias especies han sido introducidas (a veces naturalizadas) a través de plantaciones en el sur de México, Guatemala, Antillas Mayores, en las regiones tropicales de África (Madagascar), India, China y el Sudeste Asiático (Taylor, et al., 2014). Actualmente, $C$. pubescens Vahl, es considerada una planta invasora en las islas Galápagos, Hawái y Tahití, causando daños biológicos significativos a la vegetación original (Jäger, 2015).

El género es primordialmente andino, encontrándose en los bosques de montaña desde los 1000 a 3000 m. C. barbacoensis H. Karst., C. calisaya Wedd. y C. micrantha Ruiz \& Pav. son especies que también se encuentran en tierras bajas, la primera en la región del Pacífico de Colombia y Ecuador (10-1600 m), la segunda en Bolivia (200-400 m) y la tercera, menos frecuente, se ha observado en $400 \mathrm{~m}$, en Perú. La otra especie de tierra bajas, fue $C$. amazonica Standl., un taxón presente en bosques húmedos macrotérmicos de la cuenca amazónica de Brasil (Acre, Amazonas, Roraima), Colombia, Ecuador, Perú y el escudo Guayanés en Venezuela (Aymard, et al., 1990; Taylor, et al., 2004, ver Figura 1). Sin embargo, Andersson (1995), observó en $C$. amazonica Standl. varias características contrastantes o anómalas para Cinchona (e.j. corola campanulada, blanca, con ausencia de adelgazamiento, la pubescencia muy densa en el interior del tubo y en la parte externa de los lóbulos), como consecuencia creó el género Cinchonopsis $(C$. amazónica (Standl.) L. Andersson).

Las angiospermas representan el primer grupo de organismos que han sido reclasificados a través de numerosos estudios moleculares y análisis filogenéticos, basados en 
Tabla 2. Especies del género Cinchona actualmente aceptadas, con su respectiva distribución geográfica (fide Taylor (2016); Delprete \& Cortés-Ballén, 2016; Ulloa-Ulloa, et al., 2017: Ulloa-Ulloa, et al., 2018 onwards). Información en negritas y las fechas de publicación de las especies, fue incorporada por el autor.

\begin{tabular}{|c|c|c|c|c|c|c|}
\hline \multirow[t]{2}{*}{ Especies } & \multicolumn{6}{|c|}{ Países / Regiones } \\
\hline & Bolivia & América Central & Colombia & Ecuador & Perú & Venezuela \\
\hline Cinchona antioquiae L. Andersson (1998) & & & $\mathrm{X}$ & & & \\
\hline Cinchona anderssonii C. D. Maldonado (2017) & $\mathrm{X}$ & & & & & \\
\hline Cinchona asperifolia Wedd. (1848) & $\mathrm{X}$ & & & & & \\
\hline Cinchona barbacoensis H. Karst. (1859) & & & $\mathrm{X}$ & $\mathrm{X}$ & & \\
\hline Cinchona calisaya Wedd. (1948) & $\mathrm{X}$ & $\mathbf{X}$ & $\mathbf{X}$ & & $\mathrm{X}$ & \\
\hline Cinchona capuli L. Andersson (1994) & & & $\mathbf{X}$ & $\mathrm{X}$ & $\mathbf{X}$ & \\
\hline Cinchona carabayensis Wedd. $(1848)=$ C. calisaya Wedd. & $\mathbf{X}$ & & & & $\mathrm{X}$ & \\
\hline Cinchona fruticosa L. Andersson (1998) & & & & $\mathbf{X}$ & $\mathrm{X}$ & \\
\hline Cinchona glandulifera (Ruiz) Ruiz \& Pav. (1802) & & & & & $\mathrm{X}$ & \\
\hline Cinchona govana Miq. $(1861)=$ C. pubescens Vahl & & & & & $\mathrm{X}$ & \\
\hline Cinchona hirsuta Ruiz \& Pav. (1799) & $\mathrm{X}$ & & & $\mathbf{X}$ & $\mathrm{X}$ & \\
\hline Cinchona lancifolia Mutis (1793) & & & $\mathrm{X}$ & $\mathrm{X}$ & & $\mathrm{X}$ \\
\hline Cinchona lucumifolia Pav. ex Lindl. (1838) & & & & 1 & & \\
\hline Cinchona macrocalyx Pav. ex DC. (1829) & $\mathrm{X}$ & & & $\mathrm{X}$ & $\mathbf{X}$ & \\
\hline Cinchona micrantha Ruiz \& Pav. (1799) & $\mathbf{X}$ & & & & $\mathrm{X}$ & \\
\hline Cinchona mutisii Lamb. (1821) & & & & $\mathrm{X}$ & $\mathbf{X}$ & \\
\hline Cinchona nitida Ruiz \& Pav. (1799) & & & & & $\mathrm{X}$ & \\
\hline Cinchona officinalis L. (1753) & $\mathbf{X}$ & & $\mathbf{X}$ & $\mathrm{X}$ & $\mathrm{X}$ & \\
\hline Cinchona parabolica Pav. (1859) & & & & $\mathrm{X}$ & $\mathbf{X}$ & \\
\hline Cinchona pitayensis (Wedd.) Wedd. (1849) & & & $\mathrm{X}$ & $\mathrm{X}$ & $\mathrm{X}$ & \\
\hline Cinchona pubescens Vahl (1790) & $\mathrm{X}$ & $\mathrm{X}$ & $\mathrm{X}$ & $\mathrm{X}$ & $\mathrm{X}$ & $\mathrm{X}$ \\
\hline Cinchona pyrifolia L. Andersson (1998) & & & & & $\mathrm{X}$ & \\
\hline Cinchona scrobiculata Bonpl. (1808) & & & & $\mathbf{X}$ & $\mathrm{X}$ & \\
\hline Cinchona villosa Pav. ex Lindl. (1838) & $\mathrm{X}$ & & & $\mathrm{X}$ & $\mathrm{X}$ & \\
\hline
\end{tabular}

secuencias plastídicas rbcL y atpB (Angiosperm phylogeny Group: APG IV, 2016). A través de las actualizaciones del "Angiosperm phylogeny Group" se han sugerido y realizado numerosos cambios a nivel de familias, subfamilias, tribus y géneros. Entre los más los aceptados por la gran mayoría de los botánicos, están la separación de Licania en siete géneros (Chrysobalanaceae; Sothers, et al., 2016), la inclusión de las especies de Buchenavia en Terminalia (Combretaceae; Maurin, et al., 2017), y las de Tetragastris en Protium (Burseraceae; Daly \& Fine, 2018; Daly, 2019). La tribu Cinchoneae y sus géneros no ha sido exentos a estos cambios a través de filogenias. Al presente, utilizando la información de dos análisis filogenéticos, la tribu Cinchoneae es considerada un grupo monofilético sensu stricto. Esta asociación también incluye los géneros Cinchona, Cinchonopsis,
Joosia, Ladenbergia, Remijia y Stilpnophyllum (Andersson \& Antonelli, 2005; Manns \& Bremer, 2010; Taylor, 2017). Solamente, dos especies de Remijia demostraron tener afinidades inciertas dentro de este grupo y, en consecuencia, se creó el nuevo género Ciliosemina (Andersson \& Antonelli, 2005). Esté nuevo arreglo taxonómico dentro de Cinchoneae se basó en Cinchona pedunculata H. Karst. (actualmente, Ciliosemina pedunculata (H. Karst.) A. Antonelli), una especie descrita por H. Karsten en 1859 de un exsiccatum colectado por él y J. J. Triana, en la región de Susumuco, Cundinamarca en 1855 (Karsten, 1859). La otra especie, es Ciliosemina purdieana (Wedd.) A. Antonelli (basada en Remijia purdieana Wedd.), al presente endémica del valle del bajo río Magdalena, en sectores de los departamentos de Antioquia, Bolívar, y Santander. 


\section{Consideraciones finales}

La corteza de los árboles de Cinchona contiene alcaloides de la quinina (especialmente: quinina, quinidina, cinchonina y cinchonidina), los cuales se utilizaron por cerca de cuatro siglos como el único tratamiento efectivo contra la malaria (Kaufman \& Ruveda, 2005). Hasta que 1940, la cloroquina y otros compuestos antimaláricos fueron sintetizados y desarrollados (Newman, et al., 2000). Las cortezas de Cinchona calisaya Wedd. ("Quina corteza amarilla"), producen la mayor cantidad de alcaloides (Nair, 2010). Los cuatro mayores alcaloides que poseen actividades contra la malaria, tienen la particularidad, que cada uno presentan perfiles farmacéuticos diferentes (Bruce-Chwatt, 1990). Asimismo, se conoce que la corteza de otros géneros de la tribu Cinchoneae (i.g. Ladenbergia Pimentelia y Remijia), también contienen alcaloides efectivos para el tratamiento contra la malaria (Cosenza, et al., 2013). Sin embargo, la manifestación y desarrollo de nuevos linajes y cepas de Plasmodium resistentes a los tratamientos antimálaricos (Bruce-Chwatt, 1990), observados durante la guerra de Vietnam en 1965 (Trung, et al., 2004), más la ausencia de una vacuna, han permitido que la búsqueda de nuevos compuestos y tratamientos continúen. El resultado más significativo, lo representa el descubrimiento de la artemisinina, proveniente de la "Qinghaosu" (Artemisia annua L.- Asteraceae), una hierba ancestralmente utilizada en la medicina china (Tu, 2011). Este hallazgo fue galardonado con el premio Nobel de medicina en el año 2015. Por otra parte, las cantidades de alcaloides de quinina, no solo varían entre las especies, igualmente entre poblaciones de diferentes regiones, complicando la identificación de las cortezas más productivas. Las variaciones naturales de los contenidos de quinina, permanecen inexplicados, y al presente, muy pocos ensayos han estudiado este comportamiento (Maldonado, et al., 2017b).

$\mathrm{Al}$ igual que todas las regiones neotropicales, los bosques andinos con presencia de especies de Cinchona han estado sujetos a una enorme presión antropogénica en los últimos 350 años (López, 2016), a través de la sobre explotación de la corteza (actividad que en la gran mayoría de los casos produce la muerte de los árboles, fide Mendoza, et al., 2004), la deforestación y degradación. A partir del establecimiento de las grandes plantaciones de "quina" en Asia, nuevas técnicas de colectar la corteza fueron desarrolladas para evitar la mortalidad de los individuos. Entre la más utilizada está la de descortezar e inmediatamente colocar musgos que permitan regenerar la parte de la corteza afectada (Watt, 1889).

Es de hacer notar, que estos ecosistemas todavía albergar especies nuevas para la ciencia pertenecientes a la tribu Cinchoneae (Chilquillo-Torres, et al., 2017, 2019).

La amenaza de la deforestación y degradación de los bosques andinos aumenta cada día sin considerar la información científica original que ayude a la elaboración de planes de manejo, especialmente en el uso y conservación de la biodiversidad regional, la protección de los reservorios de agua, carbono y de los escenarios que apoyen en mitigar los efectos de la vegetación a los cambios climáticos. A partir del 2013, se constituyó en Dinamarca un proyecto multidisciplinario internacional conocido como "The Quest for Cinchona - A Phylogenetic Tale". Este grupo de investigación estudia la biogeografía, los alcaloides y la conservación de los bosques de Cinchona y sus taxones relacionados, que también son fuentes de la quinina en el Neotropico. Sin duda alguna, los ecosistemas andinos deben ser utilizados, pero lo racional debería ser aprovecharlos de tal forma que éstos puedan renovarse, mantener su integridad ecosistémica y persistir en el tiempo, especialmente sus bosques montanos. Sin embargo, las actividades agrícolas y pecuarias se han incrementado significativamente en la últimas cuatro décadas, eliminando su vegetación original a niveles sin precedentes. Por lo tanto, es necesario implementar un nuevo modelo de desarrollo que convivan con las actividades económicas y la conservación de los Andes. De continuar la deforestación a los ritmos actuales, se producirá una mayor pérdida de la biodiversidad, se ocasionarán cambios irreversibles en la vegetación y se presentarán una excesiva liberación a la atmosfera del $\mathrm{CO}^{2}$ previamente secuestrado y el deterioro en la calidad de vida de la población.

\section{Agradecimientos}

Se agradece, a Gustavo A. Romero (AMES) y Daniel Santamaría (MO) por la ayuda en la búsqueda de la bibliografía histórica y referencias actuales acerca del género Cinchona (Rubiaceae), en las bibliotecas de Harvard University y Louisiana State University, respectivamente. Igualmente, a Elizabeth Castañeda, José Luis Fernández (MA) Claes Persson (GB), Gustavo Romero por la revisión del manuscrito, y a Carlos Méndez por la elaboración del mapa.

\section{Referencias}

Albis, M. M. (1855). The Indians of Andaqui, New Granada. Notes of a traveler (Translated from the Spanish by J. S. Thrasher under the J.M. Vergara y Vergara \& E. Delgado supervision). Bull. Amer. Ethnological Soc. 1: 53-72.

Andersson, L. (1998). A revision of the genus Cinchona (RubiaceaeCinchoneae). Memoirs of The New York Botanical Garden. 80: $1-75$.

Andersson, L. (1997). Synopsis of the genus Ladenbergia (Rubiaceae). Nordic J. Bot. 17: 255-299.

Andersson, L. (1995). Tribes and genera of the Cinchoneae complex (Rubiaceae). Ann. Missouri Bot. Gard. 82: 409-427.

Andersson, L. (1994). Tribe Cinchoneae. Pp. 3---82 in: Rubiaceae, part. 2, G. Harling. \& L. Andersson (eds.), Flora of Ecuador, vol. 50. Berlings, Arlöv.

Andersson, L. \& A. Antonelli. (2005). Phylogeny of the tribe Cinchoneae (Rubiaceae), its position in Cinchonoideae, and description of a new genus, Ciliosemina. Taxon. 54: 17-28.

Andersson, L. \& C. Persson. (1991). Circumscription of the tribe Cinchoneae (Rubiaceae) - a cladistic approach. Pl. Syst. Evol. 178: 65-94. 
Antonelli, A., J. A. Nylander, C. Persson, C. \& I. Sanmartín. (2009). Tracing the impact of the Andean uplift on Neotropical plant evolution. Proceedings of the National Academy of Sciences. 106: 9749-9754

APG-IV. (2016). An update of the Angiosperm Phylogeny Group classification for the orders and families of flowering plants: APG IV. Bot. J. Linn. Soc. 181 (1): 1-20.

Aymard, G., N. Cuello y A. Fernández. (1990). Observaciones sobre el hallazgo de Cinchona amazónica Standl. (Rubiaceae) en la Guayana Venezolana. Biollania. 7: 125-130.

Bruce-Chwatt, L. (1990). Cinchona and quinine: a remarkable anniversary. Interdiscipl. Sci. Rev. 15: 87-93.

Carvajal, de Fr. G. (1848). Descubrimiento del río de las Amazonas según la relación hasta ahora inédita del viaje de Francisco de Orellana. (edited J. T. Medina). Imp. E. Rasco, Sevilla.

Chilquillo-Torres, E. A., A. O. Simões \& J. Albán-Castillo. (2019). Ladenbergia siranensis (Rubiaceae: Cinchoneae), a new species from the Sira Mountains, Eastern Andes of Central Peru, and the identity of Ladenbergia acutifolia. Brittonia. 71 (2): 166-171.

Chilquillo-Torres, E. A., A. O. Simões, J. Albán-Castillo \& C. M. Taylor. (2017). A New Species of Ladenbergia (Cinchoneae: Rubiaceae) from an Andean Tepui in Northeastern Peru. Systematic Botany. 42 (4): 1-4.

Coronas-Urzúa, G. (1995). El léxico de la lengua Andaqui. Filología y Lingüística. 21: 79-113.

Cosenza, G. P., N. S. Somavilla, C. W. Fagg \& M. G. Brandão. (2013). Bitter plants used as substitute of Cinchona spp. (quina) in Brazilian traditional medicine. $J$. Ethnopharmacol. 149: 790-796.

Crawford, M. J. (2016). The Andean wonder drug: Cinchona bark and imperial science in the Spanish Atlantic 1630--1800. The University Pittsburgh Press, Pittsburgh, PA. 336 p.

Cuvi, N. (2011). The Cinchona Program (1940-1945): Science and imperialism in the exploitation of a medicinal plant. Dynamis. 31 (1): 183-206.

Daly, D. C. \& P. V. A. Fine. (2018). Generic limits re-visited and an updated sectional classification for Protium (tribe Protieae). Studies in Neotropical Burseraceae XXV. Brittonia. 70: 418-426.

Daly, D. C. (2019). A new identity for Tetragastris panamensis. Studies in neotropical Burseraceae XXIX. Brittonia. 71 (3): 345-346.

Delprete, P. G. \& R. Cortés-Ballén. (2016). Rubiaceae. 2: $2252-$ -2343. In R. Bernal-González, S. R. Gradstein \& M. Celis (eds.) Cat. Pl. \& Líq. Colombia. Universidad Nacional de Colombia (Sede Bogotá), Instituto de Ciencias Naturales, Bogotá, Colombia.

Díaz-Piedrahita, S. (2010). Historia de La Medicina, Las Quinas en el Mundo y en Colombia. https:/encolombia. $\mathrm{com} / \mathrm{medicina} / \mathrm{revistas}-\mathrm{medicas} / \mathrm{academedicina/va62/}$ academ25262-quinas/

Friede, J. (1946a). Los Andaki. Revista Hist. (Pasto). 2: 6-316.

Friede, J. (1946b). Algunas consideraciones de carácter geográfico y lingüístico sobre los Andaki. Revista Hist. (Pasto). 3: $12-35$

García-Barriga, H. (1975). Flora medicinal de Colombia. Vol. III. Instituto de Ciencias Naturales. Imprenta. Nacional. Bogotá, Colombia, $495 \mathrm{p}$.

Hodge, W. H. (1948). Wartime Cinchona procurement in Latin America. Economic Botany. 2 (3): 229-257.
Jaramillo, A. J. (1951). Estudio crítico acerca de los hechos básicos en la historia de la quina". Revista de la Academia Colombiana de Ciencias Exactas, Físicas y Naturales, 30: 61-128.

Jäger, H. (2015). Biology and Impacts of Pacific Island Invasive Species. 11. Cinchona pubescens (Red Quinine Tree-Rubiaceae). Pacific Science. 69 (2): 133-153. doi. org/10.2984/69.2.1

Kaufman, T. S. \& E. A. Ruveda. (2005). The quest for quinine: those who won the battles and those who won the war. Angew. Chem. Int. Ed. Engl. 44: 854-885.

Karsten, H. (1859). Florae Columbiae (Terranumqüe adiacemtüm; specimina selecta). Tomus Primus. Apud Ferdinandi düemmlerl süccessores, Berolini, 308 p.

Kirkbride Jr, J. H. (1982). The Cinchona species of José Celestino Mutis. Taxon. 31 (4): 693-697.

La Condamine, C. M. de la. (1745). Relation Abrégée d'un Voyage fait dans l'Intériéur de l'Amérique Méridionale, Depuis la Côtes de la Mer du Sud, jusqu'aux Côtes du Brésil \& de la Guyane, en Descendant la Riviere des Amazones. Veuve Pissot, Paris.

La Condamine, C. M de la. (1738). Sur l'arbre du Quinquina. Mém. Acad. Roy. Sci. (Paris) 1738: 226-246.

Linnaeus, C. (1753). Cinchona. Species Plantarum vol. 1. Salvius. Stockholm, 172p.

Loayza-O., K., B H. de Oliveira, E. Cóndor C. \& V. Reyna P. (2010). Estudio químico de los tallos de Cinchona pubescens. Revista del Encuentro Cientifico Internacional 7 (1): 10-24.

López, N. (2016). Evaluación del paisaje y recursos escénicos después de 350 años de explotación de la "cascarilla", Cinchona officinalis L. (Rubiaceae) en el sector CajanumaRumishitana, Ecuador. Arnaldoa. 23 (2): 461-474.

Maldonado, C., C. Persson, J. Alban, A. Antonelli \& N. Rønsted. (2017a). Cinchona anderssonii (Rubiaceae), a new overlooked species from Bolivia. Phytotaxa. 297 (2): 203-208.

Maldonado, C., C. J. Barnes, C. Cornett, E. Holmfred, S. H. Hansen, C. Persson, A. Antonelli \& Nina Rønsted. (2017b). Phylogeny predicts the quantity of antimalarial alkaloids within the iconic yellow Cinchona bark (Rubiaceae: Cinchona calisaya). Frontiers in Plant Science 8 (article 391). doi: 10.3389/fpls.2017.00391

Manns, U. \& B. Bremer. (2010). Towards a better understanding of intertribal relationships and stable tribal delimitations within Cinchonoideae s.s. (Rubiaceae). Molecular phylogenetics and evolution. 56: 21-39.

Maurin, O., J. Gere, M. van der Bank \& J. S. Boatwright. (2017). The inclusion of Anogeissus, Buchenavia and Pteleopsis in Terminalia (Combretaceae: Terminaliinae). Bot. J. Linn. Soc. 184: 312-325.

Mendoza H., B. Ramírez y L. C. Jiménez. (2004). Rubiaceae de Colombia. Guía ilustrada de géneros. Instituto de Investigación de Recursos Biológicos Alexander von Humboldt. Bogotá, Colombia. 351p.

Nair, K. P. (2010). The Agronomy and Economy of Important Tree Crops of theDeveloping World. Amsterdam: Elsevier.

Newman, D. J., G. M. Cragg \& K. M. Snader. (2000). The influence of natural products upon drug discovery. Nat. Prod. Rep. 17: 215-234.

Pérez Arbeláez, E. (1947). Plantas utiles de Colombia. Editoria Victor Hugo, 3o edición, Bogotà, Colombia. 831 p. 
Prendergast, H. D. V. (2001). Jesuit's bark (Cinchona-Rubiaceae) and other medicines. Economic Botany. 55 (1): 3-6.

Rivet, P. (1924). La langue Andaki. J. Soc. Americanistes. 16: 99-110.

Rodriguez-Nozal, R. (1993). La "oficina de la flora americana" (1788-1835) y la marginación del proyecto de las expediciones botánicas ilustradas. Facultad de Farmacia, Universidad Complutense, Tesis Doctoral, Madrid. 542 p.

Roersch van der Hoogte, A. \& T. Pieters. (2015). Science, industry and the colonial state: a shift from a German-to a Dutch-controlled cinchona and quinine cartel (1880-1920). Hist. Techno. 31: 2-36.

Röhl, E. (1944). Hermann Karsten, 1817-1908: Dr. Fil. y Med. y Prof. de Botánica. 1817-1908. Boletín de la Academia de Ciencias Físicas, Matemáticas y Naturales. 10 (8): 991-1027.

Sandoval-B. Y. y C. Echendia-C. (1987). La historia de la quina desde una perspectiva regional. Colombia, 1850-1882. Anuario Colombiano de Historia Social y de la Cultura, Bogotá. 35 p.

Simón, P. (1861). The expedition of Pedro de Ursua \& Lope de Aguirre in search of El Dorado and Omagua in 1560-1 (translated from Fray P. Simón sixth historical notice of the conquest of Tierra Firme). W. Bollaert, Esq., F.R.G.S. London, $307 \mathrm{p}$.

Soares-Ferreira Jr. W., M. P. Cruz, L. Lima dos Santos, M. Franco \& T. de Medeiros. (2012). Use and importance of quina (Cinchona spp.) and ipeca (Carapichea ipecacuanha (Brot.) L. Andersson): Plants for medicinal use from the 16th century to the present. Journal of Herbal Medicine. 2 (4): 103-112.

Sothers, C. A., G. T. Prance \& M. W. Chase. (2016). Towards a monophyletic Licania: a new generic classification of the polyphyletic Neotropical genus Licania (Chrysobalanaceae). Kew. Bull. 71: 57-69.

Steere, W. C. (1945). The botanical work of the Cinchona missions in South America. Science. 101 (2616): 177-178.

Taylor, C.M. (2016). Cinchoneae. Available from: http://www. tropicos.org (Revisado: Octubre 2019)
Taylor, C. M. (2017). Another taxonomic review of the Neotropical genus Joosia (Rubiaceae, Cinchoneae). Novon. 25 (2): 214-237.

Taylor, C. M., B. E. Hammel \& D. H. Lorence. (2014). Rubiaceae. En: Manual de Plantas de Costa Rica. Vol. VII. B. E. Hammel, M. H. Grayum, C. Herrera \& N. Zamora (eds.). Monogr. Syst. Bot. Missouri Bot. Gard. 129: 464-779.

Taylor, C. M., J. A. Steyermark, P. G. Delprete, A. Vincentini, R. Cortés, D. C. Zappi, C. H. Persson, C. B. Costa \& E. Anunciação. (2004). Rubiaceae. Flora of Venezuela Guayana. 8: 497-847. Missouri Botanical Garden, USA

Trung, H.D., W. Van Bortel, T. Sochanta, K. Keokenchanh, N. T. Quang, L. D. Cong \& M Coosemans. (2004). Malaria transmission and major malaria vectors in different geographical areas of Southeast Asia. Trop. Med. Int. Health. 9: 230-237.

Tu, Y. (2011). The discovery of artemisinin (qinghaosu) and gifts from Chinese medicine. Nat. Med. 17: 1217-1220.

Ulloa Ulloa, C., P. Acevedo-Rodríguez, S. G. Beck, M. J. Belgrano, R. Bernal, P. E. Berry, L. Brako, M. Celis, G. Davidse, S. R. Gradstein, O. Hokche, B. León, S. León-Yánez, R. E. Magill, D. A. Neill, M. H. Nee, P. H. Raven, H. Stimmel, M. T. Strong, J. L. Villaseñor Ríos, J. L. Zarucchi, F. O. Zuloaga \& P. M. Jørgensen. (2018 Onwards]. An integrated Assessment of Vascular Plants Species of the Americas (Online Updates). http://tropicos. org/projectwebportal.aspx?pagename $=$ Home\&projectid $=8$ 3(consultada: Octubre, 2019)

Ulloa Ulloa, C., P. Acevedo-Rodríguez, S. G. Beck, M. J. Belgrano, R. Bernal, P. E. Berry, L. Brako, M. Celis, G. Davidse, S. R. Gradstein, O. Hokche, B. León, S. León-Yánez, R. E. Magill, D. A. Neill, M. H. Nee, P. H. Raven, H. Stimmel, M. T. Strong, J. L. Villaseñor Ríos, J. L. Zarucchi, F. O. Zuloaga \& P. M. Jørgensen. (2017). An integrated assessment of vascular plants species of the Americas. Science. 358: 1614-1617.

Watt, G. (1889). A dictionary of the economic products of India. Vol. II, cabbage to Cyperus. Government of India, Central Printing Office, Calcutta. 